\title{
Bullying e Aspectos Psicossociais: Estudo Bibliométrico
}

\author{
Francesca Stephan ${ }^{1}$ \\ Núcleo de Estudos em Violência e Ansiedade Social da Universidade Federal \\ de Juiz de Fora, Juiz de Fora, Brasil \\ Adriana Aparecida de Almeida \\ Fellipe Soares Salgado \\ Luciana Xavier Senra \\ Lélio Moura Lourenço \\ Programa de Pós-Graduação em Psicologia da Universidade Federal de Juiz de Fora, \\ Juiz de Fora, Brasil \\ Núcleo de Estudos em Violência e Ansiedade Social da Universidade Federal \\ de Juiz de Fora, Juiz de Fora, Brasil
}

\section{Resumo}

O Bullying tem sido estudado em todo o mundo por diversos seguimentos acadêmicos. O fenômeno consiste em agressões intencionais e contínuas entre pares escolares nas modalidades física, verbal ou psicológica. É protagonizado por um ou mais agressores, com a intenção de causar danos e prejuízos a um indivíduo ou a um grupo percebido como frágil, com características específicas e sem condições de se defender ou de reverter a situação. Discussões sobre as implicações do bullying são essenciais mediante a necessidade de conhecimento, prevenção e intervenção sobre este fenômeno para diferentes segmentos de atuação profissional. O presente estudo teve como objetivo identificar e analisar as publicações indexadas em diferentes bases de dados, através de revisões bibliométricas e de análise de conteúdo, que tratassem do bullying e de cinco fatores a ele associados: violência doméstica (VD), gestão educacional, criminalidade, ansiedade social e programas de intervenção antibullying. Dentre os resultados verificou-se: 66 artigos sobre VD, 25 sobre gestão educacional, 25 relativos à criminalidade, 67 referentes à ansiedade social em adultos e 165 sobre programas de intervenção. Quanto aos programas de intervenção, foram constatados indicadores de efetividade como: (a) capacitação dos professores para intervir (51), (b) a conscientização sobre aspectos relacionados ao bullying (43) e (c) o suporte promovido para os alunos individual ou coletivamente (39). Observou-se que todas as temáticas pesquisadas relacionaram-se significativamente com o bullying, o que as ressalta como fundamentais para a inovação de técnicas de intervenção e estratégias de prevenção junto ao contexto em que o fenômeno se manifesta.

Palavras-chaves: Bullying, aspectos psicossociais, programas de intervenção.

\section{Bullying and Psychosocial Aspects: Bibliometric Study}

\section{Abstract}

Bullying has been worldwide studied by different academics fields. The phenomenon is shaped by intentional and continuous assault made by peers in school. The aggression is physical, verbal or psycholo-

Endereço para correspondência: Núcleo de Estudos em Violência e Ansiedade Social, Departamento de Psicologia, Centro de Psicologia Aplicada, Rua Santos Dumont, 214, Granbery, Juiz de Fora, MG, Brasil 36010-400. E-mail: francesca_st@yahoo.com.br, adrianalmeidapsi@yahoo.com.br, fellipe.salgado@yahoo. com, senra.1x@gmail.com e leliomlourenco@gmail.com 
gical. There may be one or more bullies with the intention of causing damage or injury to an individual or to a group perceived as fragile, with specific characteristics and unable to defend themselves or to invert the situation. Discussions on the impact of bullying are essential for prevention, intervention and knowledge about this phenomenon for different segments of professional action. This study aimed to identify and analyze the publications indexed in different databases through bibliometric study and content analysis, addressing the bullying associated with five factors: domestic violence, educational managemant, crime, social anxiety and intervention programs antibullying. Among the results: 66 on domestic violence, 25 related to school management, 25 on the crime, 67 about social anxiety in adults and 165 related to intervention programs. As for intervention programs have been identified as indicators of effectiveness: (a) the training of teachers to intervene (51), (b) awareness of issues about the phenomenon (43) and (c) support to students individually or collectively (39). It was observed that all researched topics are related significantly with bullying, showing their strength in linking with innovation techniques of intervention and prevention strategies in a given context.

Keywords: Bullying, psychosocial aspects, intervention programs.

\section{Acoso Escolar e Aspectos Psicosociales: Estudio Bibliométrico}

\section{Resumen}

La intimidación o acoso escolar se ha estudiado en todo el mundo por los diversos sectores académicos. El fenómeno consiste en la agresión física, verbal o psicológicas, intencional y continuo entre los estudiantes. Puede haber uno o más acosadores con la intención de causar daño o perjuicio a una persona o a un grupo percibida como frágil, con características específicas y que no pueden defenderse o revertir la situación. Las discusiones sobre las implicaciones de la intimidación son esenciales por la necesidad de conocimiento, prevención e intervención sobre este fenómeno para los diferentes segmentos de actuación profesional. Este estudio tuvo como objetivo identificar y analizar las publicaciones indexadas en bases de datos diferentes a través de estúdio bibliométrico y análisis de contenido que abordar el acoso y los cinco factores asociados a ella: la violencia doméstica (VD), administración de la educación, la delincuencia, la ansiedad social y programas de intervención para la intimidación. Entre los resultados se encontró: 66 artículos sobre VD, 25 de gestión educativa, 25 de la criminalidad, 67 relacionada con la ansiedad social en adultos y 165 en programas de intervención. Sobre a los programas de intervención, se observaron como indicadores de efectividad: (a) la formación de los docentes para intervenir (51), (b) el conocimiento de los temas relacionados con el acoso (43) y (c) el apoyo promovido individuale o colectivamente a los estudiantes (39). Se observó que todos los temas de investigación son relacionados significativamente con la intimidación, lo que destacó como fundamental para la innovación en las técnicas de intervención y estrategias de prevención con el contexto en el que el fenómeno se manifiesta.

Palabras clave: Acoso, aspectos psicosociales, programas de intervención.

O bullying é um fenômeno comum ao ambiente escolar, que ocorre entre crianças e adolescentes envolvendo agressões física, verbal ou psicológica, intencionais e contínuas. É protagonizado por um ou mais agressores, com objetivo de causar danos e prejuízos a alguém ou a um grupo percebidos como frágeis e com características físicas, socioeconômicas, étnicas e de orien-tação sexual específicas, em situações em que a vítima não tem condições de se de- fender ou reverter à situação (Costa \& Pereira, 2010; Olweus, 1993; Senra, Lourenço, \& Pereira, 2011).

O fenômeno tem sido considerado um fator de impacto prejudicial ao desenvolvimento biológico e psicossocial dos envolvidos, o que tem gerado uma grande preocupação acerca da violência e agressividade nas escolas por parte dos educadores, pais e pesquisadores. Os prejuízos destacam-se em problemas fisiológicos 
como desordens alimentares e cardíacas, transtorno do sono e enxaquecas, além dos problemas de conduta, baixa autoestima, transtornos de humor e de ansiedade e desorganização das referências pessoais e institucionais das pessoas envolvidas (Centers of Disease Control and Prevention, 2011; Piedra, 2006; Senra, Lourenço, \& Pereira, 2011; Silva, Oliveira, Lamas, \& Barbosa, 2011).

Dentre as diversas variáveis relacionadas ou associadas ao bullying são evidenciadas neste trabalho algumas das mais prevalentes: (a) o fenômeno da violência doméstica exposto direta e indiretamente às crianças e adolescentes (Baldry, 2003; Krug, Dahlberg, Mercy, Zwi, \& Lozano, 2002; Sani, 2008; Senra, Lourenço, \& Pereira, 2011); (b) os processos de gestão educacional (Farrington \& Ttofi, 2009; Lourenço, Pereira, Paiva, \& Gebara, 2009; Lourenço, Pereira, \& Senra, 2012; Salgado \& Lourenço, 2012); (c) o envolvimento com criminalidade (Bandeira, 2009; Calhau, 2008; Fox, Elliott, Kerlikowske, Newman, \& Christeson, 2000; Lopes, 2005; Olweus, 2011a) e (d) a ansiedade social como repercussão clínica do bullying (Gladstone, Parker, \& Malhi, 2006; Hamilton, Newman, Delville, \& Delville, 2008; McCabe, Miller, Laugesen, Antony, \& Young, 2010; Tavares, 2011; Tavares $\&$ Lourenço, 2010). No que se refere a tais temáticas, destacam-se os estudos das relações sociais e interpessoais, comportamentos, atitudes e crenças que podem favorecer e propiciar outros fatores de risco ou proteção, dependendo da qualidade das relações no ambiente considerado, ao desenvolvimento biológico e psicossocial dos envolvidos (Ogden, 2004; Saforcada, Lellis, \& Mozobancyk, 2010).

Nesse sentido, compreender, sobretudo os aspectos psicossociais e de saúde correlacionados e associados ao bullying requer o conhecimento de informações obtido através de pesquisas de revisão de literatura por meio de estudo bibliométrico, que auxiliem no desenvolvimento e na promoção de estratégias de intervenção, bem como de espaços no ambiente educacional mais saudáveis ao desenvolvimento de crianças e adolescentes, os principais personagens e atores das situações de bullying (Costa, Pereira, Simões,
\& Farenzena, 2011; Sabadini, Sampaio, \& Koller, 2009; Senra, Lourenço, \& Almeida, 2011).

Considerando a importância de uma revisão sistemática da literatura sobre o tema dos fatores ou aspectos psicossociais associados ou relacionados ao bullying para o delineamento de estratégias de intervenção e prevenção, o presente estudo teve por objetivo conhecer e avaliar a produção escrita das pesquisas sobre o bullying com seus respectivos aspectos psicossociais correlatos, sobretudo, (a) violência doméstica, (b) gestão educacional, (c) criminalidade, (d) ansiedade social e (e) programas de intervenção antibullying; para compreensão de como essas variáveis mais prevalentes, associadas e correlacionadas ao fenômeno, tem sido difundidas na produção acadêmica e científica.

O bullying, assim como toda forma de violência, é um fenômeno de causalidade complexa e de múltiplas formas de manifestações (Ministério da Saúde, 2005). Os aspectos referidos neste estudo necessitam de uma investigação unificada por evidenciarem o contexto multidimensional do bullying (Tavares, 2011). As variáveis estudadas referem-se ao contexto doméstico e familiar que representa o âmbito que assegura suporte emocional e primeira socialização de crianças e adolescentes; ao processo de administração escolar, que por sua vez deve permitir ou possibilitar a ampliação das redes de socialização e suporte social além da aquisição de conhecimento; à vivencia em comunidade que, quando circunscrita pela criminalidade, pode se tornar um fator prejudicial desencadeado pelo envolvimento, muitas vezes, precoce com atividades ilícitas; ao desempenho pessoal, pois quando acometido por um transtorno de ansiedade como a ansiedade social, o indivíduo, em médio e longo prazo, apresenta comprometimento dos relacionamentos interpessoais, das atividades laborais e familiares; e, por fim, aos seguimentos profissionais e assistenciais, os quais, na tentativa de redução ou eliminação dos prejuízos e problemas ocasionados pelo bullying, tem buscado traçar medidas de solução por meio de programas de intervenção (Bandeira, 2009; Pereira, 2008; Senra, Pereira, \& Lourenço, 2011; Tavares \& Lourenço, 2010). A associação dos fatores psicossociais 
descritos e o estudo unificado dos mesmos são importantes por permitirem um entendimento mais completo dos fatores dinâmicos e interligados envolvidos no fenômeno bullying.

\section{Método}

A presente pesquisa foi realizada mediante uma busca eletrônica de artigos indexados em diferentes bases de dados, procurando identificar publicações que avaliassem os aspectos psicossociais correlacionados e associados ao bullying: (a) violência doméstica, (b) gestão educacional, (c) criminalidade, (d) ansiedade social e (e) programas de intervenção.

A análise das publicações relativas ao tema proposto foi baseada nas abordagens de pesquisa quantitativa e qualitativa, as quais são perspectivas complementares quando se tem por finalidade a aproximação, o conhecimento e o estudo de determinadas realidades, principalmente, quando considerados aspectos comuns entre elas (Reveles \& Takahashi, 2007).

A pesquisa quantitativa foi feita para investigação da frequência das publicações a partir de variáveis como: base de dados nas quais foram selecionados os artigos, autoria, nacionalidade, periódico, metodologia do estudo, os anos das publicações, entre outras. A pesquisa qualitativa se desenvolveu pela técnica de análise de conteúdo (Bardin, 2010), com a qual puderam ser analisados os principais resultados identificados nos artigos sobre as temáticas da associação ou correlação entre violência doméstica e bullying e dos programas de intervenção antibullying.

As bases eletrônicas de dados utilizadas nas buscas foram: Periódicos Capes, PsycInfo, Medline, Lilacs, Eric, Pubmed, BVS, RedaLyc, Web of Science, Dialnet, Scielo, com uso de descritores nos idiomas português e/ou inglês, o qual foi incluído por ser comum aos dicionários de termos dessas bases. Através da leitura prévia do título e dos resumos, foi possível incluir os estudos que continham os descritores utilizados para busca, enquanto que foram excluídos aqueles inacessíveis via periódicos Capes, repetidos, os livros e capítulos, e as teses e dissertações. Dessa forma, selecionaram-se as publicações com acesso ao texto completo, as quais, por sua vez, foram lidas na íntegra e analisadas com relação ao ano de publicação, autoria, periódico, base de dados, tipo de estudo, quem foram os participantes da pesquisa, como se deu a coleta dos dados e quais os principais resultados encontrados relativos à temática.

\section{Etapas para Coleta e Análise de Dados}

Bullying e Violência Doméstica (VD). A busca para o estudo da associação entre Bullying e VD foi feita nas bases Web of Science, Medline, Dialnet, Redalyc e PsycInfo, associando os termos bullying domestic violence e bullying violence, no período de 2005 a 2011 e com os referidos critérios de inclusão e exclusão. Estas bases de dados foram escolhidas de maneira a se obterem publicações oriundas dos mais diversos países. Foi observada a frequência por base de dados, autor, país, periódico, ano da publicação, metodologia, resumo, título; e foram analisados os aspectos da relação entre o contexto de VD e papel exercido no bullying, demonstrados nos resultados das publicações, tais como: agressor, vítima, vítima-agressora, todos os grupos, não especificação ou sem relação direta; e tipos de prejuízos desencadeados às crianças e adolescentes quando há associação ou correlação entre violência doméstica e bullying.

Bullying e Gestão Educacional. A busca eletrônica para o estudo da relação entre bullying e gestão educacional foi feita nas bases ERIC, PsyInfo, BVS, RedaLyc e PubMed. Nesta etapa, a recuperação de artigos foi feita através dos seguintes descritores: bullying, educador, gestão educacional, school management, school climate, school environment. Foram selecionados os artigos publicados entre 2001 e 2011 e foram observados os periódicos e ano de publicação, metodologia utilizada e as categorias referentes às percepções dos educadores referentes ao processo de gestão da escola, tendo em vista sua importância estratégica (Farrington \& Ttofi, 2009). Nota-se que fatores de proteção/risco para o comportamento dos alunos estão condicionados às normas, às expectativas e às atitudes dos educadores, juntamente com a gestão do ambiente escolar (Abramovay \& Rua, 2002; Marturano, Linhares, \& Loureiro, 2004; Mazer, Dal Bello, \& Bazon, 2009). 
Bullying e Criminalidade. Para esse estudo foi realizada uma busca eletrônica de artigos indexados nas bases de dados BVS, Dialnet, Redalyc, Web of Science, PsycInfo e Pepsic, entre os anos de 2000 e 2011. Foi utilizado o descritor bullying no campo 'título' de cada base, associado aos descritores delinquency, delinquente behaviour e crime.

Bullying e Ansiedade Social. Para o estudo da relação entre bullying na infância e ansiedade social na idade adulta, foi realizada uma busca eletrônica de artigos indexados nas bases de dados, Medline, Web of Science, PsycINFO, no período de 2005 a 2011, a partir do cruzamento das palavras-chave: bullying, social phobia e social anxiety. Foram analisados artigos que tiveram como alvo a população adulta e, depois de verificado esse critério de inclusão, a frequência por: metodologia, ano de publicação, periódico, autoria principal e temática estudada nos artigos.
Bullying e Programas de Intervenção. A busca eletrônica para o levantamento das publicações sobre intervenções para situações de bullying foi feita nas bases Web of Science, PsycInfo, Redalyc, Dialnet e Eric, associando o termo bullying aos termos intervention well faire e management well faire.

\section{Resultados}

\section{Bullying e Violência Doméstica}

A busca eletrônica para esse estudo possibilitou catalogar 401 publicações. Entretanto, com os critérios de exclusão estabelecidos, foram selecionados 66 artigos que tratavam da relação entre bullying e VD no período de 2005 a 2011. Com relação à produção anual, observou-se que os anos de 2009 e 2010 foram os de maior número de publicações. No que se refere às indexações por países, os Estados Unidos (36,2\%), Portugal $(17,7 \%)$ e Brasil $(11,2 \%)$ se destacaram com os maiores percentuais de produção (Tabela 1).

Tabela 1

Frequência de Produção de Publicações por Ano e País (n=66)

\begin{tabular}{lccccc}
\hline Ano & $N$ & $\%$ & País & $n$ & $\%$ \\
\hline 2005 & 4 & 6,06 & EUA & 24 & 36,2 \\
2006 & 5 & 7,58 & Portugal & 12 & 17,7 \\
2007 & 5 & 7,58 & Brasil & 7 & 11,2 \\
2008 & 11 & 16,67 & Espanha & 6 & 9,1 \\
2009 & 21 & 31,82 & Holanda & 5 & 7,6 \\
2010 & 12 & 18,18 & Reino Unido & 4 & 6,1 \\
2011 & 8 & 12,12 & Itália & 2 & 3,1 \\
& & & Colômbia & 1 & 1,5 \\
& & & Canadá & 1 & 1,5 \\
& & & França & 1 & 1,5 \\
& & & Chile & 1 & 1,5 \\
& & & Alemanha & 1 & 1,5 \\
& & & Suíça & 1 & 1,5 \\
\hline
\end{tabular}

O Journal of School Violence (6) e School Psychology Quarterly (5) apresentaram o maior número de publicações. O Journal of School
Violence é um periódico dedicado ao relato de pesquisas e intervenções realizadas no ambiente escolar. O School Psychology Quarterly abarca 
as publicações da divisão de psicologia escolar da Associação Americana de Psicologia e é direcionado para a divulgação de pesquisas empíricas neste contexto.

Os demais periódicos tiveram cada um, cinco, três, duas ou uma publicação. No que se referem às autorias, aqueles que foram mais frequentes nas publicações foram: (a) Bowes, L., Arseneault, L., Maughan, B., Taylor, A., Caspi, A. e Moffitt, T. E. (8,9\%); (b) Freire, I. P., Simão, A. M. V. e Ferreira, A. S. (6,7\%); (c) Finkelhor, D., Turner, H., Ormrod, R. e Hamby, S. L. (6,7\%); (d) Turner, H. A., Finkelhor, D. e Ormrod, R. (6,7\%); (e) del Rey, R. e Ortega, R. (6,7\%), (f) Martínez, J. M. A. e Mascarenhas, S., (6,7\%); e (g) Bauer, N. S., Herrenkohl, T. I., Lozano, P., Rivara, F. P., Hill, K. G. e Awkins, J. D. com $4,4 \%$ do total de publicações.

A metodologia mais utilizada e relatada nas publicações foi a qualitativa (43,9\%), com diversas técnicas (análise de conteúdo e grupos focais, por exemplo) seguida pela quantitativa com delineamento transversal para levantamento de prevalência $(22,7 \%)$ acerca da temática envolvida.

No que concerne aos resultados divulgados pelos artigos a respeito da relação entre VD e bullying, foi constatado que, dentre as crianças e/ou adolescentes vítimas diretas e/ou indiretas de VD, 2,2\% delas tornaram-se agressoras (bullies), 15,6\% eram vítimas e 35,6\% eram simultaneamente bullies e vítimas. Além disso, $17,8 \%$ das publicações analisadas mostraram que vítimas de VD foram bullies em alguma situação, vitimizadas em outras e, simultaneamente, agressora e vítima tanto de bullying quanto de VD. Por fim, 13,3\% dos artigos não relataram qualquer relação direta entre a violência doméstica e o bullying; e 15,6\% não especificaram a relação.

Quanto aos prejuízos desencadeados pela associação entre bullying e VD, foram verificados problemas psicológicos e fisiológicos $(27,3 \%)$ ou exclusivamente problemas psicológicos $(21,2 \%)$. Além desses problemas, foram relatados os de cunho psicológico e social em 16,7\% das publicações e em 13,6\% simultaneamente os problemas físicos, psicológicos e sociais. Dentre os problemas fisiológicos, $12, \%$ dos artigos destacavam dores de cabeça, desordens alimentares e cardíacas, transtornos do sono e consumo de drogas e álcool. Entre os problemas sociais e de conduta mais comuns, $7,6 \%$ das publicações relataram movimentos corporais tensos, choro, comprometimento das relações interpessoais e das habilidades sociais, repetição intencional das condutas agressivas e violentas e queda no desempenho acadêmico e escolar.

\section{Gestão Educacional/Escolar e Bullying}

Nesta etapa foram recuperadas 76 publicações permanecendo 25 para análise. Com relação à produção anual, observou-se a seguinte indexação: 2003(2); 2006(1); 2007(2); 2008(4); 2009(5); 2010(9) e 2011(2). Publicações de 2001, 2002, 2004 e 2005 não correspondiam ao objetivo do estudo. Os anos de 2009 e 2010 foram mais frequentes (56\% somados), porém observa-se um aumento gradual de pesquisas a partir de 2006.

A revista Educational Research (12\%) e Journal of School Psychology (12\%) foram as mais prevalentes. Nota-se que as revistas pesquisadas são em sua maioria do seguimento educacional/escolar e que o interesse pelo tema envolve psicólogos, educadores e outros profissionais.

A metodologia prevalente foi a correlacional (57\%), seguida por surveys $(28 \%)$ e experimental/quase-experimental $(16 \%)$. O uso de questionários diversos ocorreu em $28 \%$ das publicações. Com relação aos autores, observou-se uma variedade de pesquisadores não sendo significativa a prevalência de um sobre o outro. Esse aspecto pode ser resultado de pequeno número de artigos recuperados. Foi observada grande frequência de professores $(60 \%)$ comparada à outros funcionários da escola e diretores que juntos somam apenas $28 \%$.

A maioria dos estudos (56\%) utilizou o conceito de clima escolar (percepção de segurança, apoio e pertença, suporte de professores, regras claras de convívio) que incentivam a inclusão de toda a escola, família ou comunidade no processo de gestão.

Observou-se que os educadores sustentam crenças diferentes quanto ao sexo (gênero) dos alunos e seu envolvimento com o bullying em 
$32 \%$ das publicações. Um exemplo é a tendência em acreditar que o bullying direto do tipo físico praticado pelos meninos é comum ao período de desenvolvimento; denotando menor probabilidade de intervenção. Segundo Farrington e Ttofi (2009) em seu estudo sobre intervenção em bullying, os educadores e principalmente, os professores são centrais na prevenção e diminuição de do bullying. Sendo importante observar suas concepções sobre essa agressividade e como elas podem interferir na forma de intervir no contexto escolar.

Os níveis de clareza na definição do bullying dada pelos educadores estavam presentes em $52 \%$ dos estudos. Abarca-se aqui tanto o que é considerado bullying pelos educadores quanto a coerência de ações entre eles quando na intervenção. $\mathrm{O}$ desconhecimento, por exemplo, de outros tipos de bullying mais difíceis de detectar como o cyberbullying e o bullying indireto não permitem uma coerência em tais ações podendo gerar uma sensação de desconforto e indiferença nos alunos (Olweus, 1993; Pereira, 2008; Stelko-Pereira \& Williams, 2012).

\section{Criminalidade e Bullying}

De acordo com os critérios de inclusão, foram recuperados e analisados na íntegra $25 \mathrm{pu}-$ blicações referentes à relação entre o bullying escolar e criminalidade, entre os anos de 2000 e 2011. No que se refere a publicação anual, 2011 obteve o maior número de artigos indexados (11), seguido de 2007 com 3 e 2008 com 2. Os anos de 2010, 2009, 2006, 2005, 2004, 2003, 2001 e 2000 tiveram uma publicação cada e no ano de 2002 não foi encontrada nenhuma indexação.

Com relação aos periódicos, os que mais indexaram artigos sobre o tema foram Criminal Behaviour and Mental Health (9) e Archives of Pediatrics \& Adolescent Medicine (3). Os outros periódicos publicaram apenas um artigo cada.

Os autores mais frequentes nas publicações, em ordem alfabética, foram Farrington, D. P., Nansel, T. R., Overpeck, M. D. e Ruan, J. com três artigos cada, seguido pelos autores Elonheimo, H., Kim, M. J., Kumpulainen, K., Loeber, R., Lösel, F., Moilanen, I., Piha, J., Rönning, J.
A., Scheidt, P., Sourander, A., Tamminen, T., Ttofi, M. M. e Van der Geest, V. com duas publicações.

Com relação ao tipo de estudo, o mais utilizado foi o transversal com 13 publicações, o longitudinal com 11 e uma revisão da literatura. Em $58 \%$ dos estudos foram realizadas as pesquisas com estudantes; $12,5 \%$ com crianças e $12,5 \%$ com adolescentes selecionados na população geral, em hospitais ou que davam entrada em algum programa; $8,3 \%$ com adultos (do exército, por exemplo) e $8,3 \%$ com pessoas condenadas por algum crime. Os dados foram coletados através de questionários em $50 \%$ dos artigos; entrevistas em 17,6\%; autorrelatos em 14,7\%; registros criminais em $14,7 \%$ e observação em $2,9 \%$.

Em relação aos resultados divulgados pelos artigos a respeito da relação existente entre criminalidade e bullying, em 56\% das publicações foi encontrada associação positiva entre agressores de bullying e criminalidade; em $32 \%$ foi relatada relação positiva entre estas duas variáveis, mas sem especificar o papel exercido nos episódios de bullying; $8 \%$ encontraram relação apenas para agressores-vítimas e 4\% não encontraram nenhuma associação. Com relação às vítimas de bullying, 32\% dos artigos não encontraram nenhuma associação, $28 \%$ não analisaram as vítimas e apenas $8 \%$ acharam alguma relação.

\section{Bullying e Ansiedade Social}

Foram encontrados duzentos e quarenta e seis (246) artigos nos bancos de dados do Periódicos Capes, com os descritores bullying e social phobia ou social anxiety. Com o objetivo de investigar a relação entre bullying na infância e ansiedade social na idade adulta, foram analisados mais profundamente os 67 que tiveram como alvo a população dessa faixa etária especificamente.

Com relação à metodologia dos artigos que tratavam de população adulta, $86,5 \%(58)$ deles foram classificados como estudos empíricos e quantitativos; $10,5 \%$ (7) caracterizaram-se como estudos qualitativos; $1,5 \%$ (1) foi classificado como estudo empírico, quantitativo e qualitativo; enquanto somente $1,5 \%$ (1) artigo foi classificado como empírico, longitudinal, qualitativo 
e quantitativo. É importante salientar que esta classificação é fornecida pelos próprios bancos de dados quando descrevem sua metodologia. Quanto ao ano de publicação dos artigos, foi observada a seguinte frequência: 2008 (15), 2006 (14), 2007 (13), 2005 (13), 2009 (10) e 2010 (2). Nenhuma publicação tendo como alvo a população adulta foi publicada em 2011.

Com relação ao periódico de publicação dos artigos, observamos pouca repetição de revistas. Somente os periódicos Archives of General Psychiatry, International Journal of Mental Health, Journal of Family Violence, Nursing Research, Occupational Medicine Journal, Social Psychiatry and Psychiatric Epidemiology tiveram duas publicações cada sobre a temática no período pesquisado. Os demais periódicos tiveram somente uma publicação.
No que se refere à autoria, Sonnis, J., produziu dois artigos como primeiro autor, assim como Solomom, Z., também com dois artigos. Os demais autores publicaram somente um artigo como primeiro autor no período estudado.

Quanto ao tema dos estudos, as sessenta e sete pesquisas realizadas com a população adulta foram categorizadas da seguinte forma: estudos envolvendo o transtorno de stress pós-traumático $(n=15)$, estudos sobre violência contra o idoso $(n=3)$, estudos sobre propostas de serviços de atendimentos ou intervenções práticas $(n=5)$, estudos sobre violência contra a mulher $(n=13)$, estudos sobre sintomas físicos ou psicológicos $(n=12)$, violência no local de trabalho $(n=14)$, violência familiar contra crianças $(n=1)$, sintomas em pacientes esquizofrênicos $(n=3)$ e diferença de gênero e sintomas $(n=1)$, como demonstra a Tabela 2.

\section{Tabela 2}

\section{Temas Estudados nos Artigos}

\begin{tabular}{lcc}
\hline Temas Estudados & $N$ & $\%$ \\
\hline Transtorno de Stress Pós-traumático & 15 & 22,50 \\
Violência no local de trabalho & 14 & 21 \\
Estudos sobre violência contra a mulher & 13 & 19,5 \\
Estudos sobre sintomas físicos ou psicológicos & 12 & 18 \\
Estudos sobre violência contra o idoso & 3 & 4,5 \\
Estudos sobre serviços de atendimentos ou intervenções práticas & 5 & 7,5 \\
Sintomas em pacientes esquizofrênicos & 3 & 4,5 \\
Violência familiar & 1 & 1,50 \\
Diferença de gênero e sintomas & 1 & 1,50 \\
Total & 67 & 100 \\
\hline
\end{tabular}

\section{Programas de Intervenção}

A busca eletrônica para o estudo sobre os programas de intervenção para as situações de bullying catalogou 689 publicações. Com a utilização dos critérios de exclusão, foram selecionados 165 artigos. No que se refere à publicação anual, o ano de maior produção sobre a temática foi o de 2010 com 30,9\% do total de artigos. Na sequência, os percentuais de publicações por ano foram, respectivamente, 2009 (18,2\%),
2008 (15,8\%), 2011 (15,2\%), 2007 (10,3\%) e 2006 com 9,7\%. Em relação à publicação por países no período de 2006 a 2011, a Espanha teve $33,9 \%$ do total de artigos publicados, seguida pelos Estados Unidos (23,6\%) e Portugal $(10,9 \%)$. O Brasil, entretanto, ainda é pouco representativo nas publicações sobre intervenção para situações de bullying (3,6\%).

Para a análise de conteúdo dos resultados dos 165 artigos selecionados foi eleito como cri- 
tério inicial de leitura (Fase I) a identificação de indicadores efetivos dos programas de intervenção para situações de bullying relatados nas publicações. Com esse critério foi feita a leitura integral dos resultados e discussão de cada estudo selecionado. Por meio da leitura foram criadas as seguintes categorias de análise (Fase II): (a) conscientização sobre o bullying e as variáveis a ele relacionadas; (b) capacitação ou desenvolvimento de habilidades nos professores; (c) suporte aos alunos e (d) parceria com a comunidade e com a família.
Com a análise da categorização dos resultados e discussões das publicações foi constatado que os referidos indicadores mais frequentes referiam-se à capacitação ou desenvolvimento de habilidades dos professores para o enfrentamento do contexto de bullying em ambiente escolar $(30,9 \%)$ e à conscientização a respeito do fenômeno e das variáveis a ele relacionadas $(26,1 \%)$. Os menos citados foram o suporte aos alunos $(23,6 \%)$ e a parceria com a comunidade e a família $(19,4 \%)$ conforme pode ser observado na Tabela 3.

Tabela 3

Análise de Conteúdo dos Resultados de Discussões dos 165 Artigos Selecionados

\begin{tabular}{|c|c|c|c|c|}
\hline Fase I & \multicolumn{4}{|c|}{ Eleição do critério inicial de análise } \\
\hline Critério de leitura & \multicolumn{4}{|c|}{ Identificação dos indicadores de efetividade dos Programas antibullying } \\
\hline Fase II & Categoria 1 & Categoria 2 & Categoria 3 & Categoria 4 \\
\hline Categorização & $\begin{array}{l}\text { Conscientização } \\
\text { sobre bullying } \\
\text { e a variáveis } \\
\text { a ele vinculadas }\end{array}$ & $\begin{array}{l}\text { A preparação, } \\
\text { capacitação ou o } \\
\text { desenvolvimento } \\
\text { de habilidades } \\
\text { dos professores }\end{array}$ & $\begin{array}{c}\text { Suporte } \\
\text { aos alunos }\end{array}$ & $\begin{array}{l}\text { Parceria com } \\
\text { a comunidade } \\
\text { e com a família }\end{array}$ \\
\hline$N$ & 43 & 51 & 39 & 32 \\
\hline$\%$ & $26,1 \%$ & $30,9 \%$ & $23,6 \%$ & $19,4 \%$ \\
\hline
\end{tabular}

\section{Discussão}

Os estudos acerca do bullying têm abrangido variáveis que auxiliam na compreensão das tipologias de agressão, nos modos de vitimização e prejuízos ou impactos desencadeados na vida dos envolvidos. Entender melhor a relação entre o bullying e a violência doméstica, por exemplo, permite apreender um ciclo de violência ao qual crianças e adolescentes podem estar expostos. Uma interface com a gestão escolar permite entender melhor a realidade das escolas envolvidas com planejamento de ações que possam deter o bullying. Os impactos gerados pelo fenômeno devem ser melhor entendidos, na tentativa de ampliar o conhecimento sobre as consequências dos atos violentos na criminalidade a médio e longo prazo, e na socialização adulta, quando incapacita laboral e emocionalmente os envolvidos.

\section{Violência Doméstica e Bullying}

No que concerne à associação entre violência doméstica e bullying, a pesquisa permitiu verificar um aumento de estudos com essa temática, os quais ressaltam a importância da identificação dos prejuízos dessa relação no desenvolvimento de crianças e adolescentes e para melhorar o conhecimento do contexto que a envolve. Além disso, devem visar ao desenvolvimento de estratégias de intervenção que cessem a situação de violência na família e de bullying e vitimização no contexto escolar.

Nos estudos de prevalência sobre a referida associação, como, por exemplo, o de Bauer et al. (2006), realizado com 112 indivíduos de ambos os sexos, com idades entre 6 a 13 anos, verificou-se que $61 \%$ das crianças estavam em situação de bullying, 55\% em situação de vitimização e, destes, $97 \%$ dos bullies foram também expostos 
à violência por parceiros íntimos (Intimate Partner Violence, expressão utilizada no inglês). É interessante destacar que o objetivo geral desses estudos, através da utilização da Escala Tática de Conflitos-2 (CTS-2), foi o de identificar o bullying e a vitimização em crianças que foram expostas à violência por parceiros íntimos.

\section{Gestão Educacional e Bullying}

As publicações sobre gestão do espaço escolar em situações de bullying apresentaram fatores complexos que merecem atenção de pesquisadores, como, por exemplo, o impacto das ações de diretores e outros educadores escolares na prevenção e promoção de ambiente saudável e protetivo. Ressaltaram ainda a importância de professores adequadamente preparados no processo de intervenção.

Biggs, Vernberg, Twemlow, Fonagy e Dill (2008) sustentam que sem a adequada aderência dos professores, os programas de intervenção não apresentarão efetividade. Esse tópico se revela preocupante quando se observa as condições de trabalho do professor na rede pública diante de escolas depredadas e da falta de apoio da família. Tal preocupação não se difere quando se trata de contexto privado onde não há dano patrimonial, mas sim casos de violência velados ou não divulgados, já que a relação da escola com a família e com a comunidade é marcada também pela prestação de serviços.

\section{Criminalidade e Bullying}

As publicações analisadas com relação ao bullying e criminalidade apontam uma associação entre ambos, o que merece atenção especial dos pais, professores, pesquisadores e de todos os envolvidos neste contexto. Saber identificar e intervir em contextos de bullying traz diversos benefícios a longo prazo, e a redução da criminalidade também é um benefício possível quando se previne tal fenômeno.

Os estudos sobre o bullying e sua associação com a criminalidade são recentes, sendo evidenciados a partir das publicações no ano de 2011. Dentre os artigos analisados nessa temática, vale destacar a pesquisa realizada por Dan Olweus (2011a), com agressores de bullying do sexo masculino. $\mathrm{O}$ autor avaliou os registros criminais destes em um período de oito anos, quando se encontravam na faixa etária dos 16 aos 24 anos. Os resultados mostraram que os agressores escolares estavam fortemente representados nos registros de crime. Cerca de 55\% dos autores de bullying haviam sido condenados por um ou mais crimes e até $36 \%$ haviam sido condenados por pelo menos três crimes no período analisado. A chance dos autores de bullying reincidirem no crime (terem pelo menos três condenações) foi cinco vezes maior quando comparados aos não agressores. Com relação a crimes violentos eles tiveram de 6 a 8 vezes maior probabilidade de cometerem tais crimes dos que as crianças que não eram agressoras. Como aponta este estudo, ter um padrão de comportamento de bullying representa um forte indicador de sérios problemas antissociais mais tarde na vida.

\section{Ansiedade Social e Bullying}

O levantamento sobre os artigos acerca da relação entre bullying e ansiedade social mostrou-se importante, pois alguns estudos ressaltaram diretamente a relação entre bullying e ansiedade Social. Gladstone et al. (2006) considera que a exposição ao bullying é um preditor para ansiedade social e agorafobia por desencadear altos índices de ansiedade. $\mathrm{O}$ autor destaca, no entanto, que não são todas as vítimas de bullying que desenvolvem a Fobia Social na idade adulta, mas naquelas que a desenvolvem, o transtorno é pouco estudado.

Em relação aos temas dos artigos, nos estudos em que os sujeitos da pesquisa eram adultos, foram abordadas as relações entre bullying na infância e adolescência e episódios de violência no trabalho (mobbing) na idade adulta, assim como com outros tipos de violência na idade adulta. Houve destaque para o número elevado de resultados de estudos sobre transtorno de estresse pós-traumático (TEPT) em suas manifestações físico-químicas e psicológicas. Classificado pelo DSM-IV como uma forma de ansiedade, o TEPT mostrou-se mais sistematicamente estudado do que a Fobia Social, devendo ser alvo de uma maior investigação sobre similaridades e diferenças entre os dois tipos de ansiedade. Os 
artigos encontrados como referentes à associação entre bullying na infância e adolescência e Ansiedade Social na idade adulta são de grande relevância para o melhor entendimento do tema e servirão de base para pesquisas futuras.

\section{Programas de Intervenção Antibullying}

Em relação aos programas de intervenção para situações de bullying, Martins (2009) e Stelko-Pereira e Williams (2010) ressaltaram a importância da formação e conscientização inicial de educadores favorecida por conteúdos específicos sobre o fenômeno do bullying, como por exemplo, definição, fenômenos relacionados (violência fora da escola e na família, delinquência juvenil, entre outros), características de vítimas, agressores e espectadores, possibilitando, assim, a competência pedagógica.

A capacitação de professores apontada na categoria II da Tabela 2 é discutida por Gordillo (2011) e Lopes e Saavedra (2003). Para esses autores, o processo de capacitação e desenvolvimento é fundamental para lidar com problemas comportamentais e emocionais dos alunos e para a aplicação de técnicas de resolução de conflitos e treino assertivo e de habilidades sociais entre alunos. Contudo, fatores socioeconômicos como baixos salários, sobrecarga de trabalho, saúde ocupacional e a cultura de desvalorização do professor e do processo de educação básica, violências externas à escola, tráfico de drogas, insegurança dos bairros e comunidade aonde são sediadas as escolas, podem inviabilizar tal processo (Lisboa \& Ebert, 2012; Stelko-Pereira $\&$ Williams, 2012). Importante destacar que estes aspectos concentram atenção em habilidades dos educadores. Corroborando Farrington e Ttofi (2009), o estudo de suas concepções sobre o tema se torna relevante.

No que se refere ao suporte aos alunos, Pereira (2008) e Stelko-Pereira e Williams (2010) observam que a responsabilidade do bullying não pode ser depositada somente no professor ou educador já que o fenômeno e multideterminado. Nesse sentido, a assistência aos alunos não deve visar apenas o ambiente escolar ou a relação entre pares e destes com professores, mas também o desenvolvimento de atividades lúdicas e os padrões de comportamento e atitudes familiares decorrentes de um ambiente de agressão e violência, que podem repercutir na repetição de condutas agressivas em outros contextos como a escola e a comunidade.

A parceria da escola com a família e a comunidade apontada na categoria IV de análise das publicações como um indicador de efetividade dos programas de intervenção e prevenção de bullying, embora não seja a mais frequente nesse estudo, é mencionada por Gázquez, Cangas Díaz, Fuentes e Acién (2008) como fundamental para deter as situações de bullying, sobretudo em longo prazo. De acordo com esses autores, tal parceria contribui para redução de impactos na saúde física e psicológica e para a melhoria das relações interpessoais, pois funcionam como uma importante fonte de apoio emocional e social.

\section{Considerações Finais}

É possível considerar, com base nos resultados, que uma proposta efetiva e eficaz de intervenção para as situações de bullying deve consistir numa combinação dos indicadores destacados em cada uma das temáticas abordadas no presente estudo, conforme sugerem Avilés, Irurtia, García-Lopez e Caballo (2011), Barbosa, Lourenço e Pereira (2011) e Olweus (2011b) nas investigações desenvolvidas e nos programas de intervenção já aplicados em diversos contextos em todo o mundo.

Nesse sentido, diante do que foi exposto, é possível considerar que os fatores psicossociais associados ao bullying analisados no presente estudo podem propiciar reflexões acerca de prejuízos e impactos negativos na vida de crianças e adolescentes em curto, médio e longo prazos, assim como a respeito das possibilidades de intervenção frente ao fenômeno. Essas reflexões evidenciam desde problemas na família expressados na forma de violência que tende a ser adotada e reproduzida como estratégia de resolução de conflitos e de relacionamentos interpessoais; até os problemas sociais e de saúde como o comprometimento da gestão educacional, o envolvimento em atividades ilícitas e o transtorno de an- 
siedade social, que por sua vez compromete não somente a vida pessoal de um indivíduo, mas as atividades laborais e os relacionamentos interpessoais, demandando atenção cada vez mais acentuada de diferentes seguimentos de atuação profissional.

\section{Referências}

Abramovay, M., \& Rua, M. G. (2002). Violência nas escolas. Brasília, DF: Organização das Nações Unidas para a Educação, a Ciência e a Cultura.

Avilés, J. M., Irurtia, M. J., García-Lopez, L. J., \& Caballo, V. E. (2011). El maltrato entre iguales: "bullying". Psicología Conductual, 19(1), 57-90.

Baldry, A. C. (2003). Bullying in schools and exposure to domestic violence. Child Abuse \& $\mathrm{Ne}$ glect, 27(2003), 713-732.

Bandeira, C. M. (2009). Bullying: Autoestima e diferença de gênero (Dissertação de mestrado, Universidade Federal do Rio Grande do Sul, Porto Alegre, RS, Brasil).

Barbosa, A. G., Lourenço, L. M., \& Pereira, B. O. (Orgs.). (2011). Bullying: Conhecer e intervir. Juiz de Fora, MG: Universidade Federal de Juiz de Fora.

Bardin, L. (2010). Análise de conteúdo. Lisboa, Portugal: Edições 70.

Bauer, N. S., Herrenkohl, T. I. L., Lozano, P., Rivara, F. P., Hill, K. G., \& Hawkins, D. (2006). Childhood bullying involvement and exposure to intimate partner violence. Pediatrics, 118(2), 235-242.

Biggs, B. K., Vernberg, E., Twemlow, S. W., Fonagy, P., \& Dill, E. J. (2008). Teacher adherance and its relation to teacher attitudes and student outcomes in an elementary school-based violence prevention program. School Psychology Review, 37(4), 533-549.

Calhau, L. B. (2008). Bullying, criminologia e a contribuição de Albert Bandura. Recuperado em 08 outubro de 2011, de http://www.mp.to.gov.br/ cint/cesaf/arqs/300309035719.pdf

Centers of Disease Control and Prevention. (2011). Bullying among middle school and high school students - Massachusetts, 2009. Morbidity and Mortality Weekly Report, 60(15), 465-496. Re- trieved April 22, 2011, from http://www.cdc. gov/mmwr/preview/mmwrhtml/mm6015a1. htm?s cid $=$ mm6015a 1 w

Costa, P., \& Pereira, B. O. (2010). O bullying na escola: A prevalência e o sucesso escolar. In L. Almeida, B. Silva, \& S. Caires (Orgs.), Actas do I Seminário Internacional Contributos da Psicologia em Contexto Educativo (pp. 1810-1821). Braga, Portugal: Centro de Investigação em Educação, Instituto de Educação, Universidade do Minho.

Costa, P., Pereira, B. O., Simões, H., \& Farenzena, R. (2011). Vitimação em contexto escolar: Frequência e as múltiplas formas. In B. Pereira \& G. Carvalho (Orgs.), Actas do VII Seminário Internacional de Educação Física, Lazer e Saúde: A actividade fisica promotora de saúde e desenvolvimento pessoal e social (pp. 18971912). Braga, Portugal: Centro de Investigação de Estudos da Criança, Instituto de Educação, Universidade do Minho.

Farrington, D. P., \& Ttofi, M. M. (2009). Schoolbased programs to reduce bullying and victimization. Oslo, Norway: Campbell Systematic Reviews.

Fox, J. A., Elliott, D. S., Kerlikowske, G., Newman, S. S., \& Christeson, W. (2000). Bullying prevention is crime prevention (Report 2000). Washington, DC: Fight Crime, Invest in Kids.

Gázquez, J. J., Cangas Díaz, A. J., Fuentes, M. C. P., \& Acién, F. L. (2008). Comparative analysis of the perception of school violence in teachers, pupils and families. The Spanish Journal of Psychology, 11(2), 443-452.

Gladstone, G. L., Parker, G. B., \& Malhi, G. S. (2006). Do bullied children become anxious and depressed adults? A cross-sectional investigation of the correlates of bullying and anxious depression. The Journal of Nervous and Mental Disease, 194(5), 201-208.

Gordillo, I. C. (2011). Divergence in aggressors' and victims' perceptions of bullying: A decisive factor for differential psychosocial intervention. Children and Youth Services Review, 33(9), 1608-1615.

Hamilton, L. D., Newman, M. L., Delville, C. L., \& Delville, Y. (2008). Physiological stress responses of young adults exposed to bullying during adolescence. Physiology \& Behavior, 95(5), 617-624. 
Krug, E. G., Dahlberg, L. L., Mercy, J. A., Zwi, A. B., \& Lozano, R. (Orgs.). (2002). World report on violence and health. Geneva, Switzerland: World Health Organization.

Lisboa, C., \& Ebert, G. (2012) Violência na escola: Reflexão sobre as causas e propostas de ação preventiva e focais. In L. F. Habigzang \& S. H. Koller (Orgs.), Violência contra crianças e adolescentes: Teoria, pesquisa e prática (pp. 190202). Porto Alegre, RS: Artmed.

Lopes, A. A., Neto, \& Saavedra, L. H. (2003). Diga não para o bullying: Programa de redução do comportamento agressivo entre estudantes. Rio de Janeiro, RJ: Associação Brasileira Multiprofissional de Proteção à Infância e a Adolescência.

Lopes, A. A., Neto. (2005). Bullying - Comportamento agressivo entre estudantes. Jornal de Pediatria, 81(5), 164-172.

Lourenço, L. M., Pereira, B. O., \& Senra, L. X. (2012). A gestão educacional na caracterização e na prevenção do bullying. In Actas do II Seminário Internacional Contributos da Psicologia em Contextos Educativos (pp. 365-374). Braga, Portugal: Centro de Investigação em Educação, Instituto de Educação, Universidade do Minho.

Lourenço, L. M., Pereira, B. O., Paiva, D. P., \& Gebara, C. F. P. (2009). A gestão educacional e o bullying: Um estudo em escolas portuguesas. Interacções (Lisboa), 5(13), 208-228.

Martins, M. J. D. (2009). Maus tratos entre adolescentes na escola. Lisboa, Portugal: Novembro.

Marturano, E. M., Linhares, M. B. M., \& Loureiro, S. R. (2004). Vulnerabilidade e proteção: Indicadores de trajetória de desenvolvimento do escolar. São Paulo, SP: Casa do Psicólogo.

Mazer, S. M., Dal Bello, A. C., \& Bazon, M. R. (2009). Dificuldades de aprendizagem: Revisão de literatura sobre os fatores de risco associados. Psicologia da Educação, 28(2), 7-21.

McCabe, R. E., Miller, J. L., Laugesen, N., Antony, M. M., \& Young, L. (2010). The relationship between anxiety disorders in adults and recalled childhood teasing. Journal of Anxiety Disorders, 24(2), 238-243. doi: 10.1016/j.janxdis.2009.11.002

Ministério da Saúde. (2005). Impacto da violência na saúde dos brasileiros. Brasília, DF: Autor.
Ogden, J. (2004). Psicologia da Saúde (2. ed.). Lisboa, Portugal: Climepsi.

Olweus, D. (1993). Bullying at school: What we know and what we can do. Oxford, MA: Blackwell.

Olweus, D. (2011a). Bullying at school and later criminality: Findings from three Swedish community samples of males. Criminal Behaviour and Mental Health, 21(2), 151-156.

Olweus, D. (2011b). What is bullying? Retrieved September 27, 2011, from http://www.olweus. org/public $2 \mathrm{~m}$ /bullying.page

Pereira, B. O. (2008). Para uma escola sem violência - Estudo e prevenção das práticas agressivas entre crianças (2. ed.). Lisboa, Portugal: Fundação Calouste Gulbenkian.

Piedra, R. R. (2006). Crianças contra crianças: O bullying, uma perturbação emergente. Anales de Pediatría, 1(2), 101-104.

Reveles, A. G., \& Takahashi, R. T. (2007). Educação em saúde ao osteomizado: Um estudo bibliométrico. Revista da Escola de Enfermagem da USP, 41(2), 245-250.

Sabadini, A. A. Z. P., Sampaio, M. I. C., \& Koller, S. H. (Orgs.). (2009). Publicar em psicologia: Um enfoque para a revista cientifica. São Paulo, SP: Associação Brasileira de Editores Científicos de Psicologia.

Saforcada, E., Lellis, M., \& Mozobancyc, S. (2010). Psicologia y Salud Publica - novos aportes desde la perspectiva del factor humano. Buenos Aires, Argentina: Paidós.

Salgado, F. S., \& Lourenço, L. M. (2012). Publicações referentes à gestão do ambiente escolar $e$ ao bullying. Trabalho apresentado no V Simpósio de Psicologia e Desenvolvimento Humano, IV Encontro de História e Filosofia da Psicologia, III Colóquio de Psicologia Social e Políticas Públicas, Juiz de Fora, MG, Brasil.

Sani, A. I. (2008). Crianças expostas à violência interparental. In C. Machado \& R. A. Gonçalves (Orgs.), Violência e vítimas de crimes: Crianças (pp. 95-127). Lisboa, Portugal: Quarteto.

Senra, L. X., Lourenço, L. M., \& Almeida, A. A. (2011). Bullying e violência doméstica: Considerações a partir de um estudo bibliométrico. In VI Congreso Internacional de Psicología y Educación, Valladolid-Espanha. Educación, aprendizaje y desarrollo en una sociedad mul- 
ticultural (pp. 9517-9530). Madrid, Espanha: Associación de Psicología y Educación.

Senra, L. X., Lourenço, L. M., \& Pereira, B. O. (2011). Características da relação entre violência doméstica e bullying: Revisão sistemática da literatura. Gerais: Revista Interinstitucional de Psicologia, 4(2), 297-30.

Silva, A. N., Oliveira, J. C., Lamas, K. A., \& Barbosa, A. G. (2011). Pesquisas sobre bullying no Brasil. In A. G. Barbosa, L. M. Lourenço, \& B. Pereira (Orgs.), Bullying: Conhecer e intervir (pp. 1131). Juiz de Fora, MG: Universidade Federal de Juiz de Fora.

Stelko-Pereira, A. C., \& Williams, L. C. A. (2010). Reflexões sobre o conceito de violência escolar e a busca por uma definição abrangente. Temas em Psicologia, 18(1), 41-54.

Stelko-Pereira, A. C., \& Williams, L. C. A. (2012). Dando voz a estudantes de escolas públicas sobre situações de violência escolar. In L. F. Habigzang \& S. H. Koller (Orgs.), Violência contra crianças e adolescentes: Teoria, pesquisa e prática (pp. 203-218). Porto Alegre, RS: Artmed.
Tavares, F. S. (2011). Estudo compreensivo da associação entre bullying e ansiedade social (Dissertação de mestrado, Universidade Federal de Juiz de Fora, MG, Brasil).

Tavares, F. S., \& Lourenço, L. M. (2010). Levantamento bibliométrico sobre bullying e fobia social. In Anais do I Seminário Internacional "Contributos da Psicologia em Contextos Educativos” (pp. 1461-1470). Braga, Portugal: Universidade do Minho
Recebido: 1\%10/2012

$1^{a}$ revisão: $23 / 11 / 2012$

$2^{a}$ revisão: 06/01/2013

$3^{a}$ revisão: 02/02/2013

Aceite final: 06/02/2013 